\title{
Excitation Energy Transport and Concentration Depolarization of Donor Fluorescence in Two-Component Viscous Solutions*
}

\author{
C. Bojarski ${ }^{a}$, A. Kawski $^{\text {b }}$, A. Kubicki ${ }^{\text {b }}$, and G. Żurkowska ${ }^{\text {a }}$ \\ a Institute of Physics, Technical University, Gdańsk, Poland \\ b Institute of Experimental Physics, University of Gdańsk, Gdańsk. Poland \\ Z. Naturforsch. 43a, 297-302 (1988); received January 6, 1988
}

The concentration depolarization of the fluorescence of rhodamine B (donor) in the presence of malachite green (acceptor) was investigated in a glycerol-ethanol mixture with constant ratios of the reduced concentrations of the donor and acceptor (system I: $\gamma_{\mathrm{D}} / \gamma_{\mathrm{A}}=1$, system II: $\gamma_{\mathrm{D}} / \gamma_{\mathrm{A}}=19$ ). A marked effect of the configuration of the active molecules surrounding the successively excited donors upon the emission anisotropy was found in system I, having strong fluorescence quenching and weak migration, whereas in system II, with strong migration and weak fluorescence quenching, this effect is smaller. The results are discussed based on the up-to-date theories of the concentration depolarization of fluorescence.

\section{Introduction}

Recently progress has been made in understanding the incoherent nonradiative excitation energy transfer (NET) in disordered systems of donor (D) and acceptor (A) molecules by taking into account the preferential return of the excitation energy to the molecule from which this energy has just been transferred [1-10]. Such a mechanism of NET is possible if a correlation exists between the configurations of the $\mathrm{D}$ and A molecules surrounding the excited D molecule before and after the transfer. This NET process should lower the effective migration rate, and, as a result, affect the emission anisotropy and the fluorescence quantum yield of the donor.

In the present paper, experimental results concerning the concentration depolarization of the fluorescence (CDF) of a donor in presence of an acceptor are reported. In order to investigate the effect of the correlation upon the $\mathrm{CDF}$, these results are compared with corresponding theories, with and without accounting for the correlations. Such investigations seem useful since few papers have dealt with this problem [11].

\section{Theoretical Basis}

In the case of statistical distribution of the molecular dipole directions in space, it has commonly been

* The research reported in this article was sponsored by the Polish Ministry of National Education under Project CPBP 01.06 .

Reprint requests to Prof. Dr. C. Bojarski, Politechnika Gdańska, Katedra Luminescencji Molekularnej, ul. Majakowskiego 11/12, 80-952 Gdańsk, Poland. assumed in the CDF theories that only molecules $\mathrm{D}_{i}$, excited primarily by light absorption, contribute to the observed emission anisotropy, $r,[12-14]$. In this case $[15-16]$

$$
r / r_{0}=\eta_{i} / \eta
$$

where $\eta_{i}$ is the fluorescence quantum yield of molecules $\mathrm{D}_{i}$, and $\eta$ - the total fluorescence quantum yield.

Recently, within the framework of approximation (1) the following slightly different expressions have been obtained for the emission anisotropy:

$$
\left(r / r_{0}\right)_{1}=\eta_{i} / \eta_{1}
$$

in [17] and

$$
\left(r / r_{0}\right)_{2}=\eta_{i} / \eta_{2},
$$

in [18], where

$$
\begin{aligned}
& \eta_{i}=\eta_{0}\left[1-f\left(\gamma^{\prime}\right)\right], \\
& \eta_{1}=\eta_{0}[1-f(\gamma)] /[1-\alpha f(\gamma)], \\
& \eta_{2}=\eta_{0}\left[1-f\left(\gamma^{\prime}\right)\right] /\left[1-\alpha^{\prime} f\left(\gamma^{\prime}\right)\right], \\
& f(x)=\pi^{1 / 2} x \exp \left(x^{2}\right)[1-\operatorname{erf}(x)], \\
& \gamma=\gamma_{\mathrm{D}}+\gamma_{\mathrm{A}}=2^{-1} \pi^{1 / 2}\left(\frac{C_{\mathrm{D}}}{C_{\mathrm{ODD}}}+\frac{C_{\mathrm{A}}}{C_{\mathrm{ODA}}}\right) ; \\
& \alpha=\frac{\gamma_{\mathrm{D}}}{\gamma}, \\
& \gamma^{\prime}=2^{-1 / 2} \gamma_{\mathrm{D}}+\gamma_{\mathrm{A}} ; \\
& \alpha^{\prime}=2^{-1 / 2} \gamma_{\mathrm{D}} /\left(2^{-1 / 2} \gamma_{\mathrm{D}}+\gamma_{\mathrm{A}}\right) .
\end{aligned}
$$

$C_{\mathrm{D}}, C_{\mathrm{A}}$, and $C_{0 \mathrm{DD}}, C_{0 \mathrm{DA}}$ denote the concentrations and critical concentrations of the donor (D) and the acceptor (A) molecules, respectively, $\eta_{0}$ is the absolute

0932-0784 / 88/0400-0297 \$01.30/0. - Please order a reprint rather than making your own copy. 


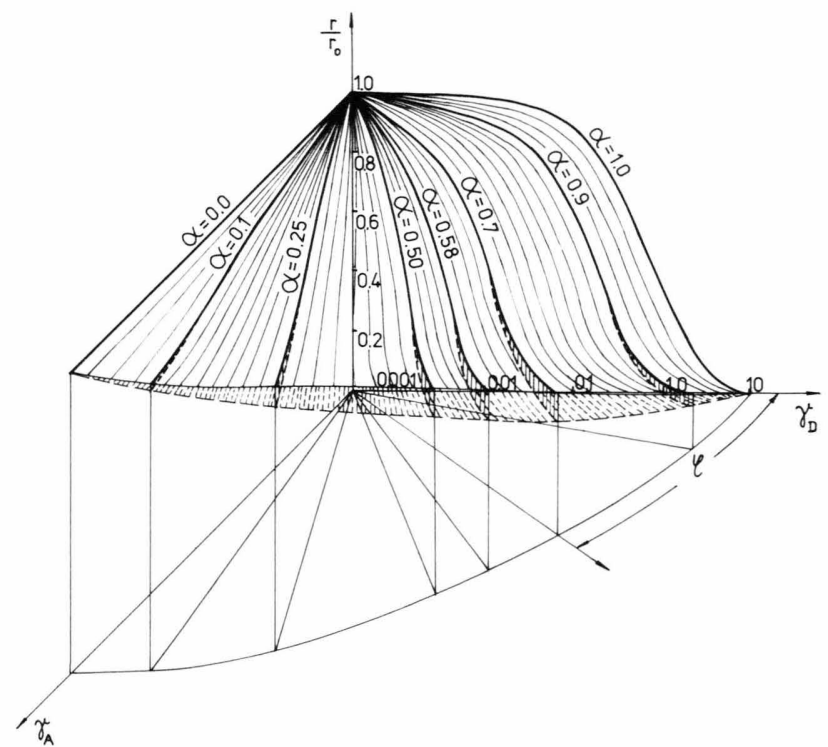

Fig. 1. Emission anisotropy, $r / r_{0}$, vs. reduced concentrations, $\gamma_{\mathrm{D}}$ and $\gamma_{\mathrm{A}}$. Solid and broken curves are the lines of the intersection of surfaces determined by (2) and (3) with the plane $\gamma_{\mathrm{A}} / \gamma_{\mathrm{D}}=\operatorname{tg} \varphi=$ const.

fluorescence quantum yield of the donor when $C_{\mathrm{D}}$ and $C_{\mathrm{A}}$ approach zero.

In (6), as opposed to (5), the correlations between the configurations of the luminescent molecules in the vicinity of successively excited donors have partly been taken into consideration. These expressions differ only in the replacement of the concentration $\gamma_{\mathrm{D}}$ with lower concentration $2^{-1 / 2} \gamma_{\mathrm{D}}$. Hence the introduction of the above correction should result in diminishing of the effective range of migration and the excitation energy trapping by the acceptors. As a result, an increase in the quantum yield and a decrease in the emission anisotropy should be expected $\left(\left(r / r_{0}\right)_{2}<\left(r / r_{0}\right)_{1}\right.$, see Fig. 1.).

The emission anisotropies given by (2) and (3) are functions of two variables, $\gamma_{D}$ and $\gamma_{A}$.

In the case of systems with constant ratio $\gamma_{\mathrm{A}} / \gamma_{\mathrm{D}}$, i.e. with a constant $\alpha=1 /\left(1+\gamma_{\mathrm{A}} / \gamma_{\mathrm{D}}\right),(2)$ and (3) are represented in Fig. 1 by solid and broken curves, respectively, resulting from the intersection of the surface $\left(r / r_{0}\right)$ $\left(\gamma_{A}, \gamma_{D}\right)$ with the plane $\gamma_{\mathrm{A}} / \gamma_{\mathrm{D}}=$ const.

For $\varphi=\operatorname{arctg}\left(\gamma_{\mathrm{A}} / \gamma_{\mathrm{D}}\right)=0$ or $\pi / 2$, the solid and broken curves coincide in the whole concentration range $\left\{\Delta r=\left(r / r_{0}\right)_{1}-\left(r / r_{0}\right)_{2}=0\right\}$, whereas $\Delta r>0$ for $\varphi \varepsilon(0, \pi / 2)$ at high concentrations.

The values of $\Delta r$ depend both on $\alpha$ and $\gamma_{\mathrm{D}}$. For the experimental investigation of the effect of the correla-

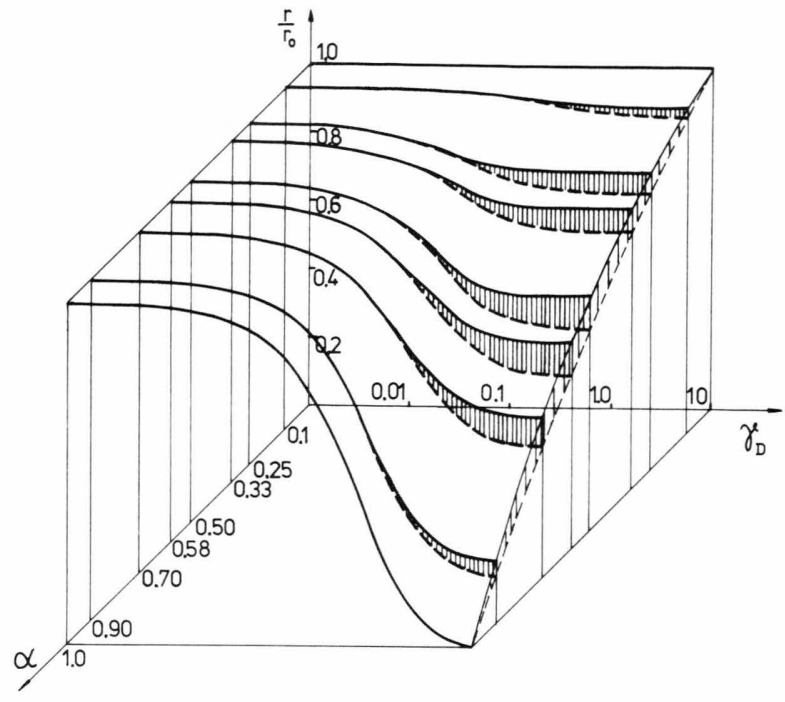

Fig. 2. Emission anisotropy vs. $\gamma_{\mathrm{D}}$ for several values of $\alpha$. Solid and broken curves were obtained according to (2) and (3). For $\gamma_{\mathrm{D}}=10$, a maximum $\Delta r=\left(r / r_{0}\right)_{1}-\left(r / r_{0}\right)_{2}=0.102 \mathrm{oc}-$ curs at $\alpha=0.586\left(\gamma_{\mathrm{A}} / \gamma_{\mathrm{D}}=2^{-1 / 2}\right)$.

tion on the emission anisotropy, the choice of a system, for which the expected differences $\Delta r$ are the highest, is essential. Let us note that for a system with constant $\gamma_{\mathrm{A}} / \gamma_{\mathrm{D}}$, the emission anisotropy $r / r_{0}$ approaches asymptotically a constant value of $\left(r / r_{0}\right)_{g}$ for $\gamma_{\mathrm{D}} \rightarrow \infty$, and the difference $\Delta r$ reaches a limiting value

$$
\begin{aligned}
\Delta r_{\mathrm{g}}= & \left(r / r_{0}\right)_{1 \mathrm{~g}}-\left(r / r_{0}\right)_{2 \mathrm{~g}} \\
& =\alpha(\alpha-1)(2-\sqrt{2}) /\left[2^{1 / 2}(1-\alpha)+\alpha\right]^{2} .
\end{aligned}
$$

In fact, already for $\gamma_{\mathrm{D}} \approx 10$ the values of $r / r_{0}$ scarcely exceed $\left(r / r_{0}\right)_{\mathrm{g}}$ even in systems with a high value of $\alpha$. Then, also the values of $\Delta r$ differ insignificantly from $\Delta r_{\mathrm{g}}$.

Figure 2 shows theoretical courses of (2) and (3) as functions of $\gamma_{D}$ for several values of $\alpha$. The differences $\Delta r_{\mathrm{g}}$, and hence also $\Delta r$, reach maximum values for $\alpha_{\max } \approx 0.586\left(\gamma_{\mathrm{A}} / \gamma_{\mathrm{D}}=2^{-1 / 2}\right)$, which is illustrated by the intersections of curves $\left(r / r_{0}\right)_{1}$ and $\left(r / r_{0}\right)_{2}$ with the plane $\gamma_{\mathrm{D}}=10$. Two donor-acceptor systems were chosen for the investigations, with $\alpha=1 / 2$ and $\alpha=0.95$, which correspond to markedly different values of $\Delta r_{\mathrm{g}}$.

\section{Experimental}

For the experimental verification of Expr. (2) and (3), two series of glycerol-alcohol (GA) mixture solutions 
of rhodamine B (donor) and malachite green (acceptor) with the values of $C_{\mathrm{D}} / C_{\mathrm{A}}=1.14(\alpha=0.5$, system I) and $C_{\mathrm{D}} / C_{\mathrm{A}}=21.63(\alpha=0.95$, system II) were prepared. Rhodamine $\mathrm{B}\left(\mathrm{C}_{28} \mathrm{H}_{31} \mathrm{O}_{3} \mathrm{~N}_{2} \mathrm{Cl}\right.$; m.w. 479.03) and malachite green $\left(\mathrm{C}_{25} \mathrm{H}_{26} \mathrm{~N}_{2} \mathrm{O}_{4}\right.$; m.w. 418.53) were purified by recrystallization. Anhydrous glycerol mixed with $10 \%$ of ethanol, both analytically pure, were used as a solvent without further purification.

Sample cells were formed by pairs of glass plates separated by thin spacers of thickness $d$ small enough for the relation $2.3 \varepsilon_{\mathrm{D}}^{\max } C_{\mathrm{D}} d<0.1$ to hold, where $\varepsilon_{\mathrm{D}}^{\max }$ is the maximum value of the donor extinction coefficient. Under these conditions, the effect of the reabsorption and secondary fluorescence on $r / r_{0}$ can be neglected [19].

The fluorescence spectra were measured upon frontal excitation and observation of the sample, and corrected for the spectral sensitivity of the photomultiplier. For the absorption measurements a VSU 2-P spectrophotometer was employed.

The emission anisotropy was measured by the photon counting method using the apparatus shown in Figure 3. Photomultipliers $\mathrm{Ph}_{1}$ and $\mathrm{Ph}_{2}$ were supplied with high voltage $U_{\mathrm{s}}=-2200 \mathrm{~V}_{\mathrm{DC}}$, yielding singlephoton pulses with an amplitude of about $-0.5 \mathrm{~V}$ and a halfwidth of about $4 \mathrm{~ns}$. The pulses were converted by the A-D discriminators (amplification, division by 8 , and ECL-TTL translation) into standard TTL pulses counted subsequently by an universal digital counter with a maximum input frequency of $50 \mathrm{MHz}$.

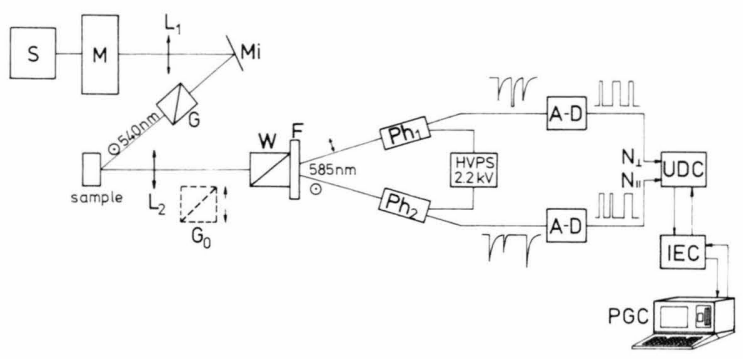

Fig. 3. Schematic diagram of the apparatus for the emission anisotropy measurements: $\mathrm{S}$-source of the exciting light, $250 \mathrm{~W}$ OSRAM Xenon Lamp; $\mathrm{M}$-monochromator, SPM-2, Carl Zeiss Jena; $\mathrm{L}_{1}, \mathrm{~L}_{2}$-lenses, Mi-mirror, $\mathrm{G}$-glan prism; $\mathrm{G}_{0}$-standard of a $r=0$ Glan prism, $\mathrm{W}$ - Wollaston prism, $\mathrm{F}$-interference and glass filters; $\mathrm{Ph}_{1}$, $\mathrm{Ph}_{2}-$ EMI $9883 \mathrm{QB}$ photon counting photomultipliers; HVPS - high voltage power supply, ZWN -41, ZZUS Polon; UDC - universal two chanel, digital counter, KZ 2026-2A, ZOPAN, Poland, IEC - IEC interface; PGC - Programmable Graphic Calculator, NK-777D, EMG Hungary; A-D amplifier-discriminator units with a TTL output constructed according to the concept presented in [20].
The whole experiment was controlled by a graphic calculator via an IEC-625 interface.

In view of low intensity of the observed light (in extreme cases amounting scarcely to 800 counts/s, with 40 counts/s for the background) it was indispensable to take into account dark photons, the photons originating from dispersion as well as some imperfections of the optical elements used (filters, Glan prisms, etc.). Moreover, due to the differing amplifications of the two photomultipliers, the value of $\varrho_{0}=I_{\perp} / I_{\|}$should be determined during each measurement for the emission anisotropy standard $r=0$. The proper by corrected value of the ratio $I_{\perp} / I_{\|}$can then be determined relative to the above value of $\varrho_{0}$. Thus, taking the above considerations into account, one obtains

$$
\begin{aligned}
& r=\frac{I_{\|}-I_{\perp}}{I_{\|}+2 I_{\perp}}=\frac{1-x}{1+2 x}, \\
& I_{\perp}=\frac{N_{\perp}-N_{\perp}^{\mathrm{d}}}{N_{0 \perp}-N_{0 \perp}^{\mathrm{d}}}, \quad I_{\|}=\frac{N_{\|}-N_{\|}^{\mathrm{d}}}{N_{0 \|}-N_{0 \|}^{\mathrm{d}}}, \\
& x=\frac{I_{\perp}}{I_{\|}}=\left(\frac{N_{\perp}-N_{\perp}^{\mathrm{d}}}{N_{0 \perp}-N_{0 \perp}^{\mathrm{d}}}\right) /\left(\frac{N_{\|}-N_{\|}^{\mathrm{d}}}{N_{0 \|}-N_{0 \|}^{\mathrm{d}}}\right)=\frac{\varrho}{\varrho_{0}},
\end{aligned}
$$

where

$$
\begin{aligned}
& \varrho=\frac{N_{\perp}-N_{\perp}^{\mathrm{d}}}{N_{\|}-N_{\|}^{\mathrm{d}}}, \quad \varrho_{0}=\frac{N_{0 \perp}-N_{0 \perp}^{\mathrm{d}}}{N_{0 \|}-N_{0 \|}^{\mathrm{d}}}, \\
& N_{\perp}^{\mathrm{d}}=n_{\perp}^{\mathrm{d}} \cdot t, \quad N_{0 \perp}^{\mathrm{d}}=n_{\perp}^{\mathrm{d}} \cdot t_{0}, \\
& N_{\|}^{\mathrm{d}}=n_{\|}^{\mathrm{d}} \cdot t, \quad N_{0 \|}^{\mathrm{d}}=n_{\|}^{\mathrm{d}} \cdot t_{0} .
\end{aligned}
$$

$N_{\perp}, N_{\|}$and $N_{0 \perp}, N_{0 \|}$ are the mean numbers of counts in respective channels for the solution investigated and for the standard $r=0$, respectively; $n_{\perp}^{\mathrm{d}}, n_{\|}^{\mathrm{d}}$ the mean numbers of counts in respective channels for the background, and $t, t_{0}$ the times of a single measurement of the two components for the solution studied and for the standard $r=0$, respectively. The indices $\perp$ and $\|$ denote the components at the output of the Wollaston prism, which are perpendicular and parallel to the polarization plane of the exciting light, respectively. The values of $n^{\mathrm{d}}, N_{0}$, and $N$ were measured simultaneously for the two channels during the same time in order to eliminate the effect of the fluctuations of the exciting light intensity.

The measurements were carried out in the following order:
1. $n_{\perp}^{\mathrm{d}}$ and $n^{\mathrm{d}}$ were measured for pure solvent replacing the sample investigated. 
Table 1. Data characterizing the system of rhodamine B and malachite green $/ \mathrm{RhB}+\mathrm{MG} /$ in a glycerol-ethanol mixture at $293 \mathrm{~K}$.

\begin{tabular}{|c|c|c|c|c|c|c|c|c|c|c|c|c|c|c|}
\hline \multirow[t]{2}{*}{$\begin{array}{l}\text { Sys- } \\
\text { tem }\end{array}$} & \multirow[t]{2}{*}{ Solvent } & \multirow[t]{2}{*}{$\frac{C_{\mathrm{D}}}{C_{\mathrm{A}}}$} & \multirow[t]{2}{*}{$\alpha$} & \multirow[t]{2}{*}{$r_{0}$} & \multirow{2}{*}{$\begin{array}{l}\text { Visco- } \\
\text { sity in } \\
\text { poise }\end{array}$} & \multirow[t]{2}{*}{$n$} & \multirow[t]{2}{*}{$\eta_{0}$} & \multirow{2}{*}{$\left\langle x^{2}\right\rangle^{*}$} & \multirow{2}{*}{\multicolumn{2}{|c|}{$\frac{C_{0 \mathrm{DD}} C_{\mathrm{ODA}}}{10^{-3} \mathrm{M}}$}} & \multirow{2}{*}{$\frac{R_{0 \mathrm{DD}}{ }^{* *}}{\AA}$} & \multirow{2}{*}{$\frac{R_{0 \mathrm{DA}}}{\AA}$} & \multirow{2}{*}{$\frac{\lambda_{\mathrm{ex}}}{\mathrm{nm}}$} & \multirow[t]{2}{*}{$\lambda_{\text {obs }}$} \\
\hline & & & & & & & & & & & & & & \\
\hline I & $\begin{array}{l}\text { glycerol }+ \\
10 \% \text { ethanol }\end{array}$ & 1.14 & 0.5 & 0.3656 & 6.38 & 1.4653 & 0.6 & 0.478 & 2.85 & 2.50 & 51.8 & 54.1 & $540 \pm 5$ & $580 \pm 5$ \\
\hline II & & 21.63 & 0.95 & 0.3566 & & & & & & & & & & \\
\hline
\end{tabular}

* A factor depending on the mutual orientation of the transition moments of the interacting molecules.

** The critical separation distance.

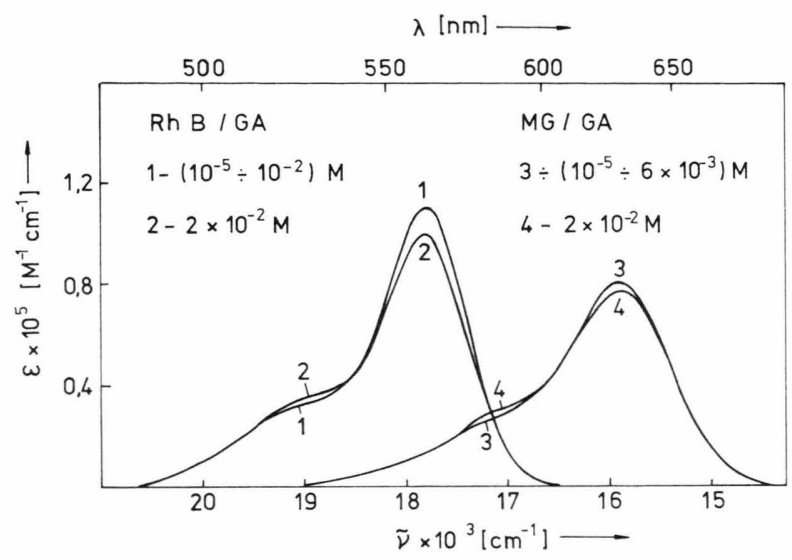

Fig. 4. Electronic absorption spectra of rhodamine B (RhB) and malachite green (MG) in glycerol-ethanol (GA) mixture.
2. $N_{0 \perp}$ and $N_{0 \|}$ were measured for the sample by inserting the Glan prism $G_{0}$ into the detection pathway and turning it by $45^{\circ}$ relative to the polarization plane of the Glan prism $\mathrm{G}\left(I_{\|}=I_{\perp}, r=0\right)$.

3. The Glan prism $G_{0}$ was removed, and the values of $N_{\perp}$ and $N_{\|}$were measured.

All quantities $n_{\perp}^{\mathrm{d}}, n_{\|}^{\mathrm{d}}, N_{0 \perp}, N_{0 \|} N_{\perp}, N_{\|}$measured in the experiment were averaged over a great number of measurements $(100 \div 1000)$. The errors were determined by their mean standard deviations. The value of the emission anisotropy was calculated according to (10)-(13), whereas the error in the emission anisotropy, $\Delta r$, was assumed to be represented by the value of the total differential of $r$, according to (10)-(13).

\section{Results and Discussion}

Figure 4 shows the electronic absorption spectra of rhodamine $\mathrm{B}(\mathrm{RhB})$ and malachite green $(\mathrm{MG})$ in glycerol-ethanol mixtures. These spectra were recorded in the concentration range from $10^{-5}$ to $2 \cdot 10^{-2} \mathrm{M}$, and found to be unchanging up to $10^{-2} \mathrm{M}$ and $6 \cdot 10^{-3} \mathrm{M}$ for rhodamine $\mathrm{B}$ and malachite green, respectively. It was therefore assumed that within those concentration ranges the presence of the ground state dimer is negligible. For $C_{\mathrm{D}}>10^{-2} \mathrm{M}$ and $C_{\mathrm{A}}>6 \cdot 10^{-3} \mathrm{M}$, a slight deformation of the spectra occurs, evidencing the formation of dimers in the solutions. The experimental values of the emission anisotropy, $r / r_{0}$, for systems I and II are shown in Figure 5. Theoretical curves a, b, c and d were calculated from expressions (2) and (3) for the values of parameters determined from independent measurements and given in Table 1. For system II with strong migration, curves $\mathrm{c}$ and $\mathrm{d}$ coincide almost in the whole concentration range, whereas for system I, where the migration
Fig. 5. Fluorescence emission anisotropy, $r / r_{0}$, of rhodamine $\mathrm{B}$ in the presence of malachite green vs. $\gamma_{\mathrm{D}}$; system I $\left(\gamma_{\mathrm{D}} / \gamma_{\mathrm{A}}=1\right), \quad$-experimental points, $\bullet$-the results of simulation; System II $\left(\gamma_{\mathrm{D}} / \gamma_{\mathrm{A}}=19\right), \times$-experimental points. 


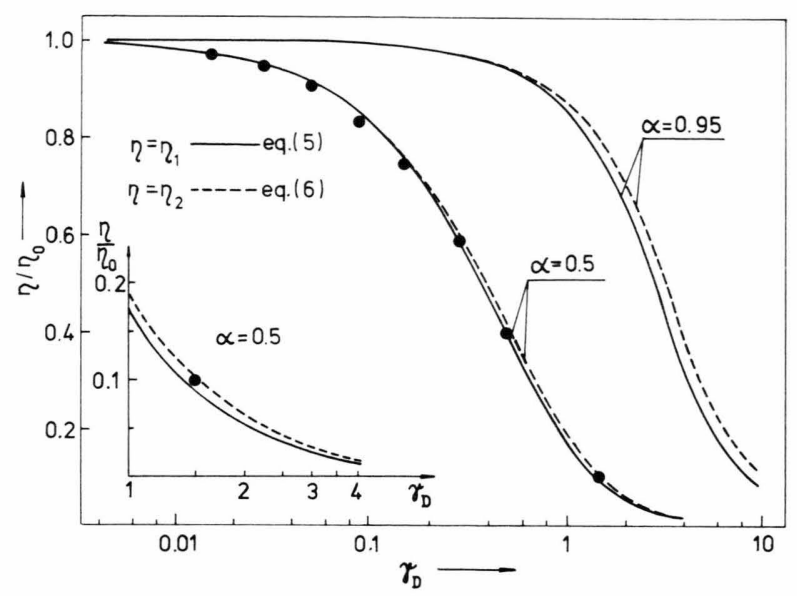

Fig. 6. Donor fluorescence quantum yield $\eta / \eta_{0}$ in the presence of the acceptor as a function of $\gamma_{D}$, obtained theoretically for systems I and II; - the results of simulation.

over donors can be neglected, the respective curves a and $b$ differ markedly. The experimental values of $r / r_{0}$ for system I fit well the curve $b$, and at high concentrations they deviate considerably from curve a; this reflects the marked effect of the correlation upon the emission anisotropy in this system. The slight scatter of the experimental points at the highest concentrations may be due to strong fluorescence quenching, as well as to the formation of dimers of molecules D and A. For system II, almost in the whole concentration range the experimental values of $r / r_{0}$ exceed slightly the theoretical values. This discrepancy may result from the derivation of expressions (2) and (3) involving the use of the averaged value of the orientation factor occurring in $R^{-6}$ - the Förster rate for the NET [21]. The calculations of $r / r_{0}$ carried out by Bodunov [22] by the use of the Monte Carlo technique involving an accurate treatment of the orientation factor yielded for unary systems the values of $r / r_{0}$ exceeding by $(10-15 \%)$ those of $r / r_{0}$ obtained for the preaveraged value of this factor. It has also been shown theoretically by other authors [23-25] that the proper approach to the orientation factor in the CDF theory leads to an increase in the emission anisotropy. It should be emphasized that the theoretical curves $a, b$, c and $\mathrm{d}$ were calculated with no adjustable parameters.

A comparison of the results presented in Fig. 5 allows for the conclusion that in systems with strong migration the effect of the correlation on the emission anisotropy can be neglected. Figure 6 shows the yield $\eta / \eta_{0}$ calculated from (5) and (6), which were obtained when neglecting (solid curve) and taking into account (broken curve) the correlation in the system. According to the anticipations, in system II with higher $\alpha$, the effect of the correlation upon the value of $\eta / \eta_{0}$ is more pronounced than it is in system I with lower $\alpha$. However, for system II the values of $\eta / \eta_{0}$ are several times higher than those for system I at the same value of concentration $\gamma_{D}$. Hence a distinct effect of the correlation upon the emission anisotropy in system I occurs, while such an effect is absent in system II, although the differences in the values of $\eta / \eta_{0}$ calculated from (5) and (6) are markedly smaller for system I (c.f. Figure 6).

Figures 5 and 6 show also the results of the simulation of the concentration depolarization and concentration quenching obtained for system I by the Monte Carlo technique [26]. The correlations between the configurations of successively excited donors were taken into account in a natural way in the simulation process. As is seen in Fig. 5, the values $r / r_{0}$ obtained from the simulation, as well as those obtained experimentally are close to curve b. In the case of the quantum yield, $\eta / \eta_{0}$, the values calculated from expressions (5) and (6), as well as those obtained by the Monte Carlo simulation coincide virtually within the whole concentration range investigated (Figure 6).

It can be concluded, based on the investigations carried out, that the effect of the correlation upon the emission anisotropy is essential only in the region of high donor concentrations and for the systems in which strong quenching of the donor fluorescence by the acceptor traps occurs $(0.2<\alpha<0.9)$.

We should like to thank Professor A. Schmillen for his helpful advice.
[1] V. L. Ermolaev, J. N. Bodunov, J. B. Sveshnikova, and T. A. Shahverdov, Non radiative Electronic Excitation Energy Transfer in Russian, Leningrad 1977.

[2] A. Kawski, Photochem. Photobiol. 38, 487 (1983).

[3] W. A. Gaysenok and A. M. Sarshevskii, Anisotropiya pogloscheniya u luminesceniji mnogoatomnych molekul, Minsk, Izd. Universitetskoje 1986.
[4] D. L. Huber, D. S. Hamilton, and B. Barnett, Phys. Rev. B 16, 4662 (1977).

[5] D. L. Huber, Phys. Rev. B 20, 5333 (1979).

[6] C. R. Gochanour, H. C. Andersen, and M. D. Fayer, J. Chem. Phys. 70, 4254 (1979).

[7] R. F. Loring, H. C. Anderson, and M. D. Fayer, J. Chem. Phys. 76, 2015 (1982). 
[8] R. Twardowski, J. Kuśba, and C. Bojarski, Chem. Phys. 64, 239 (1982).

[9] A. I. Burshtein, Zh. Exp. Theor. Phys. 84, 2001 (1983).

[10] S. G. Fedorenko and A. I. Burshtein, Chem. Phys. 98, 341 (1985).

[11] R. K. Bauer and H. Cherek, Bull. Acad. Pol. Sci. Ser. Sci. Math. Astron. Phys. 20, 961 (1972); H. Cherek, Bull. Acad. Pol. Sci. Ser. Sci. Math. Astron. Phys. 24, 135 (1976); A. Kawski and H. Szmaciński, Z. Natruforsch. 35a, 697 (1979); 37 a, 64 (1982).

[12] M. D. Galanin, Trudy Fiz. Inst. Akad. Nauk USSR 5, 341 (1950).

[13] I. Ketskeméty, Acta Phys. Chem. Szeged, 1, 29 (1955).

[14] A. Jabłoński, Acta Phys. Polon., A 38, 453 (1970).

[15] E. L. Eriksen and A. Ore, Phys. Norvegica 2, 159 (1967).

[16] C. Bojarski and J. Domsta, Acta Phys. Hung. 30, 145 (1971).
[17] R. Twardowski and C. Bojarski, J. Luminesc. 33, 79 (1985).

[18] A. I. Burshtein, J. Luminesc. 34, 201 (1985).

[19] I. Ketskeméty, J. Dombi, R. Horvai, J. Hevesi, and L. Kozma, Acta Phys. Chem. Szeged 7, 17 (1961).

[20] E. J. Darland, J. E. Hornsuh, C. G. Enke, and G. E. Leroi, Anal. Chem. 51, 245 (1979).

[21] Th. Förster, Ann. Phys. 2, 55 (1948).

[22] J. N. Bodunov, Opt. i Spektroskopiya 50, 1007 (1981).

[23] W. F. Craver and R. S. Knox, Molec. Phys. 22, 385 (1971).

[24] W. F. Craver, Molec. Phys. 22, 403 (1971).

[25] J. Knoester and J. E. Van Himbergen, J. Chem. Phys. 86, 4438 (1987)

[26] S. Błoński, K. Sienicki, and C. Bojarski, Proceedings of Intern. Symp, Molec. Luminesc. and Photophys., Torun (1986) p. 57. 